\title{
Sensor-integrated tap holder for process uncertainty detection based on tool vibration and axial length compensation sensors
}

\author{
Tuğrul Öztürk ${ }^{1}$ (D ) Erkut Sarıkaya ${ }^{1} \cdot$ Matthias Weigold $^{1}$
}

Received: 31 March 2021 / Accepted: 27 July 2021 / Published online: 13 August 2021

(C) The Author(s) 2021

\begin{abstract}
The tapping process is one of the most widespread manufacturing processes for internal threads, usually carried out at the end of the value chain. Any non-compliance with required quality standards or even the destruction of the thread due to process uncertainty in the tapping process is therefore subjected to high rework costs. Possible process uncertainties in the tapping process can be triggered by synchronization errors between feed rate and spindle speed, axis offset, faulty core holes and wear of the tapping tool. In order to detect process uncertainties during tapping and thus provide a basis for initiating countermeasures, a sensor-integrated tap holder was developed. This paper presents the realized concept of a rotating telemetry unit for signal processing, data acquisition and wireless data transmitting via WiFi standard on basis of low-cost embedded systems. Furthermore, two unique sensor concepts for measuring close-to-tool vibrations and the axial length compensation of the tapping tool are shown. Based on the sensor data in combination with feature engineering methods, process uncertainty during tapping are detected.
\end{abstract}

Keywords Tapping $\cdot$ Sensor-integrated tool holder $\cdot$ Process uncertainty $\cdot$ Feature engineering

\section{Introduction}

The manufacturing of internal threads by tapping process is common and widely used [6]. For example in the aerospace industries, where quality requirements are crucial [17]. Since the tapping process is usually carried out at the end of the value chain, any non-compliance with required thread quality or destruction of the thread due to process uncertainty is subjected with high rework costs. The machining of internal threads is one of the most complex boring operations, since an exact profile with precise dimensions and shape in narrow tolerances is required [20]. Besides the careful selection of the tapping tool in terms of design and application, one of the most crucial conditions is the synchronization between feed rate and spindle speed to achieve a constant thread pitch [13]. The occurrence of large synchronization errors while tapping can lead to a destruction of the thread while retreating [1]. In addition to synchronization errors further process uncertainties are faulty core holes, progressed wear

Tuğrul Öztürk

t.oeztuerk@ptw.tu-darmstadt.de

1 Institute of Production Management, Technology and Machine Tools, Otto-Berndt-Straße 2, 64287 Darmstadt, Germany of the tapping tool, axis offset and run-out errors [3, 10]. To master process uncertainty during tapping a semi-active control approach of the tapping process can be considered, whereby such a control requires the ability of detecting process uncertainty during the tapping process. Therefore, this paper presents a sensor-integrated tap holder consisting of a developed telemetry unit that rotates with the tap holder and two sensor concepts for process uncertainty detection during tapping. In Section 2, we will show contributions and approaches from research as well as industry for process uncertainty detection during tapping, followed by a short description of possible process uncertainties during tapping process in Section 3. In Section 4, the sensorintegrated tap holder will be introduced. We will show the experimental setup for validation experiments of the sensorintegrated tap holder in Section 5 and the signal processing of the measured data in Section 6, whereby the validation results are discussed in Section 7. The contribution ends with conclusions and gives the outlook.

\section{State of the art}

There are various approaches for process uncertainty detection during tapping. Chen et al. could detect tap tool wear, 
core hole size error and misalignment for 1/4-20-in. threads with a success rate of $95 \%$ by using an information measure and probability voting approach based on input signals acquired by a dynamometer [3]. The same results were achieved by Liu et al. by using a neural network for process uncertainty detection [11]. The research [11] was repeated in 2013 using Adaptive Neuro-Fuzzy Inference Systems (ANFIS), whereby Liu et al. showed that ANFIS structures are appropriate to distinguish a normal from an abnormal tapping process [12]. However, within the contributions of Chen et al. and Liu et al. an intrusive dynamometer was used to provide cutting forces and torque as input signals for the various approaches, which is often applicable in laboratory environment only $[3,11,12]$. Based on the motor spindle currents measured by non-intrusive Hall effect current sensors and utilizing a two-stage pair-wise feature selection and classification approach, process uncertainties such as tap wear, core hole size errors and misalignment for M10x1.25 threads could be detected by Li et al. [10]. An online monitoring and quality classification system tested with M10 size threads by using motor spindle currents and a statistical process control approach under laboratory conditions is shown in by Gil del Val et al. in [5], whereas the validation of that system in an industrial environment is presented in [19]. Furthermore, Gil del Val et al. could validate the monitoring system using the torque signal provided by the machine control [6]. Polvorosa et al. [17] detected tap wear by measuring cutting force and torque with a commercially available sensor-integrated floating tap holder (see [14]). Monka et al. could detect the wear of M12 sized tapping tools by means of frequency spectrum analysis of vibration signals measured on motor spindle and workpiece [16]. An approach based on a vision system for breakage detection of tapping tools of size down to M1 was shown by Lee et al. in [9].

Besides the scientific contributions cited here, there are three commercially available sensor-integrated tool holders for the tapping process applicable within industrial environment. The first one is the HFP System [2], developed by the company Bilz Werkzeugfabrik $\mathrm{GmbH} \& \mathrm{Co}$. KG, which detects the occurrence of axial compensation due to synchronization errors without quantifying it and thus providing a binary information only. The second system denoted as DDU Sensorsystem was developed by ARTIS MARPOSS [14]. It provides a tool breakage detection during tapping. The company pro-micron $\mathrm{GmbH}$ has developed the sensor-integrated tool holder spike which enables tapping tool wear detection for preventing tool breakage by means of axial force and bending torque measurements based on strain gauge technology [7]. At this point it should be emphasized that other process monitoring systems for tapping exist, but they do not use closeto-process-related data such as spindle or drive currents. However, the focus of the presented work is on sensorintegrated tool holders for tapping.

In this contribution we present an experimental sensorintegrated tap holder, in which two sensors and a telemetry unit are integrated. The first sensor enables the measurement of axial length compensation caused by synchronization errors and in contrast to [2] provides the quantification of the length compensation for process uncertainty analysis. The second sensor enables the measurement of low and high frequency vibrations of the tapping tool. Compared to the vibration measurement approach presented in [16], the vibration sensor shown in this work is directly connected with the tapping tool and allows to measure the vibrations in two mutually perpendicular directions. The use of a dynamometer as in the contributions [3, 11, 12] is deliberately avoided here, since it is cost-intensive and not always applicable in industrial environment. Furthermore, the use of motor spindle currents as data source for process uncertainty detection during tapping is also avoided, since this approach is restricted to large thread sizes as M10 [5, 10]. Beside the sensor technology, the approach presented here is using WiFi for wireless sensor data transmission, which differs from the wireless technologies used in $[2$, $7,14]$ and enables therefore the use of standard network technologies to upload sensor data into cloud applications for data analysis and visualization.

For the sake of completeness, it should be mentioned that several research projects already exist in the field of sensorintegrated tool holders for other machining operations such as turning and milling. The main challenges in the development of sensor-integrated tool holders can be divided into two classes, namely the scientific and technological challenges. The scientific challenges deal with the determination of appropriate measurement quantities. On the other hand, the technological challenges include among others the integration of the sensors into the tool holder with minimum interfering contours, the provision of power supply, and reliable wireless communication. However, two examples should be mentioned here: A smart cutting tool for turning operations that measures cutting and feed forces in a real machine environment based on a wireless surface acoustic wave (SAW) strain sensor is shown in [21]. Two passive SAW strain sensors were attached directly on the tool shank, whereby the transmission of the measured quantity is carried out using a wireless interrogator. A smart milling tool for monitoring face milling operation is shown in [15] which is equipped with strain gauges, a triaxial accelerometer, and thermal sensors. The power supply is provided by an integrated rechargeable battery. 


\section{Process uncertainty during tapping process}

As in other manufacturing processes, the tapping process can be afflicted with several process uncertainties which are shown in Fig. 1 and are discussed in this section, whereby process uncertainty is abbreviated to uncertainty only from now on. Axis offset occurs when the center tool axis does not coincide with the center axis of the core hole illustrated in Fig. 1a. Since the tapping process is a two-stage manufacturing process, uncertainty can occur during drilling the core holes within the first stage such as sloped drills (Fig. 1b), faulty core hole diameter (Fig. 1c) or stucked metal chips in the core holes after drilling process. Further, progressed wear of the drilling tool can lead to elliptical geometries of the core hole. Other uncertainties which can occur during the tapping stage are synchronization and runout error. A synchronization error occurs when the motor spindle speed $n$ and the feed rate in $z$-axis direction $v_{f}$ is not synchronized, thus leading to erroneous pitch $p$ of the manufactured thread (Fig. 1d). According to the definition of the thread pitch by Eq. 1, a synchronization error occurs when the ratio of feed rate $v_{f}$ in $z$-axis direction and motor spindle speed $n$ differs from the pitch of the tapping tool.

$p=\frac{v_{f}}{n}$

The occurrence of synchronization errors results from insufficient precision of the machine tool numeric control and the drives or from erroneous tapping cycle parameters. Superimposed unbalances of the tapping tool itself, the tapping tool holder and the motor spindle can lead to run-out errors. Finally, uncertainties which can lead to tapping tool breakage are progressed tool wear and trapped chips which cause jamming of the tapping tool when turning out.

The commercially available sensor-integrated tap tool holders have gaps in terms of detecting the various uncertainties in tapping. In case of synchronization errors, which can cause tapping tool breakage due to high cutting torque or influence the thread quality [1], there are no sensor-integrated tapping tool holders which can quantify synchronization errors. Furthermore, the sensor-integrated tool holders are restricted to the detection of tapping tool wear or breakage. The presented sensor-integrated tap tool holder offers the opportunity to minimize this gap in uncertainty detection, thus allowing the detection of axis offset, faulty core holes, synchronization errors as well as tapping tool breakage.

\section{Sensor-integrated tap holder}

The sensor-integrated tap holder is based on the Softsynchro 3 from the company Emuge Franken. It is a purely mechanical system for compensating synchronization errors allowing the tapping tool to move along the longitudinal axis, a so-called floating tap holder. The functional principle of the floating tap holder shown in Section 4.1 allows the detection as well as the quantification of synchronization errors by integrating the axial length compensation sensor introduced in Section 4.3. The second sensor concept for uncertainty detection is the close-to-tool vibration sensor introduced in Section 4.2. The principle of a direct sensor attachment on the tapping tool allows the measurement of vibrations close to the tool center point, thus avoiding the influence by other mechanical components of the tapping tool holder such as the mechanical interface of the linear guidance for length
Fig. 1 Types of uncertainty during tapping process: axis offset (a), sloped pre-drills (b), faulty core hole diameter (c) and synchronization error (d)

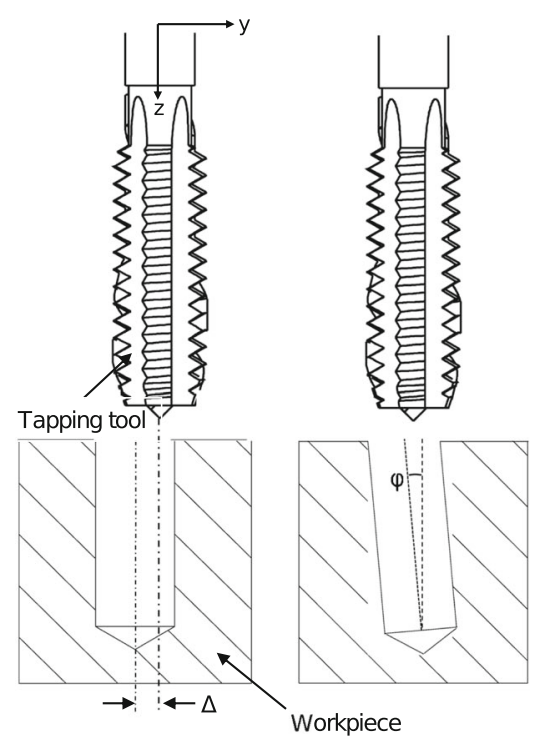

(a) (b)

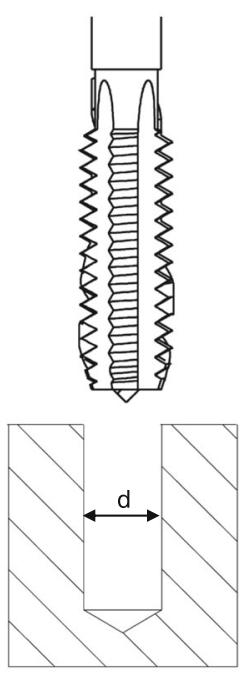

(c)

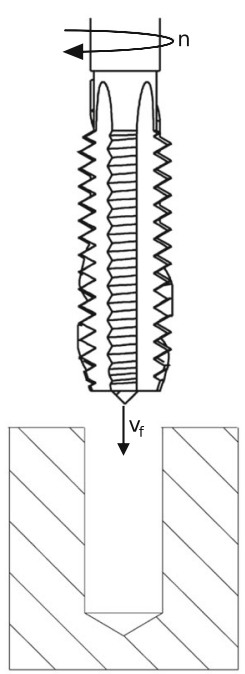

(d) 
compensation. Furthermore, this sensor concept makes the sensor-integrated tap holder more sensitive, which is essential for small tapping tool diameters. The data acquisition as well as the wireless data transmission is done by a telemetry unit which is shown in Section 4.4.

\subsection{Floating tap holder}

The Softsynchro 3 shown in Fig. 2 consists of four components: (1) an A63 type hollow shank taper, (2) a cylindrical attachment rigidly connected with the hollow shank taper, (3) a movable piston, (4) the tapping tool. The piston (3) is aligned with the $z$-axis by two rolling elements (5) which are offset by $180^{\circ}$, thus enabling a degree of freedom for the tap in both $z$-axis directions. The rolling elements (5) also prevent the rotation of the piston (3) about the $z$-axis, thus allowing the transmission of torque from the motor spindle to the tapping tool.

The stiffness in $z$-axis direction is determined by the two pairs of polymer spring elements (6), which are also offset by $180^{\circ}$. The floating tap holder allows a maximum stroke of the tapping tool of $\pm 1.5 \mathrm{~mm}$.

\subsection{Sensor for measuring close-to-tool vibrations}

For measuring the close-to-tool vibrations, a sensor concept has been developed based on capacitive Micro-ElectroMechanical System (MEMS) accelerometer sensors. The MEMS sensors used for this design provide a wide frequency bandwidth from DC up to $11 \mathrm{kHz}$ with a sensitivity of $20 \frac{\mathrm{mV}}{\mathrm{g}}$ and a measurement range of $\pm 100 \mathrm{~g}$. These specifications allow structural dynamic measurements such as tool vibrations. The developed close-to-tool vibration sensor on basis of the two MEMS accelerometers enables a direct measurement of the radial vibrations $a_{x}$ and $a_{y}$ in two perpendicular directions of the tapping tool as illustrated in Fig. 3b.

The close-to-tool vibration sensor shown in Fig. 3a consists of a housing (6) which carries the printed circuit board (5) with two uniaxial MEMS accelerometers (3 and 4). A sleeve (2) with an external thread is attached on the tapping tool (1) by an adhesive. This allows to screw the housing (6) on the sleeve (2) to establish a rigid connection between the tapping tool (1) and the housing. This type of sensor application was deliberately chosen to reuse the vibration sensor in case of tapping tool breakage.

\subsection{Sensor for measuring the length compensation}

The sensor that measures the axial length compensation of the floating tap holder is depicted in Fig. 4a. The sensor element consists of an outer (1) and inner (2) ring, which are connected by two webs (3), thus functioning as a ring spring element.

The spring element is connected with a spacer ring (5) which is connected on the bottom side (9, Fig. 2) of the cylindrical attachment (2, Fig. 2) with an adhesive. Figure $4 b$ shows the integration of the sensor into the floating tap holder, whereas Fig. 2 position (7) shows the sensor in the integrated state. In case of axial length compensation, the piston (3, Fig. 2) moves according to Fig. 2) in negative $z$ axis direction and presses the inner ring (2) of the sensor. The resulting stress within the two webs (3) yielding a strain state, which is measured by the full-bridge strain gauge (4). The screw (6), which is located at the bottom side (8, Fig. 2) of the piston (3, Fig. 2), enables the adjustment of the sensors pretension.

Since the axial length compensation sensor measures the strain, we will now discuss the conversion of measured strain to the length compensation, thus displacement. Each of the polymer spring elements (6, Fig. 2) is connected to the piston (3, Fig. 2) with a single screw only shown in Fig. 5. This allows the modelling of polymer ring elements $c_{\mathrm{p}}$ and the sensor $c_{\mathrm{s}}$ by simple spring elements as shown in Fig. 5, whereby damping effects are neglected. Considering linear elastic deformation of the sensor element, Hooke's law and first order beam theory can be utilized to gain the necessary equations.
Fig. 2 Quarter sectional view of the Softsynchro 3 [4]

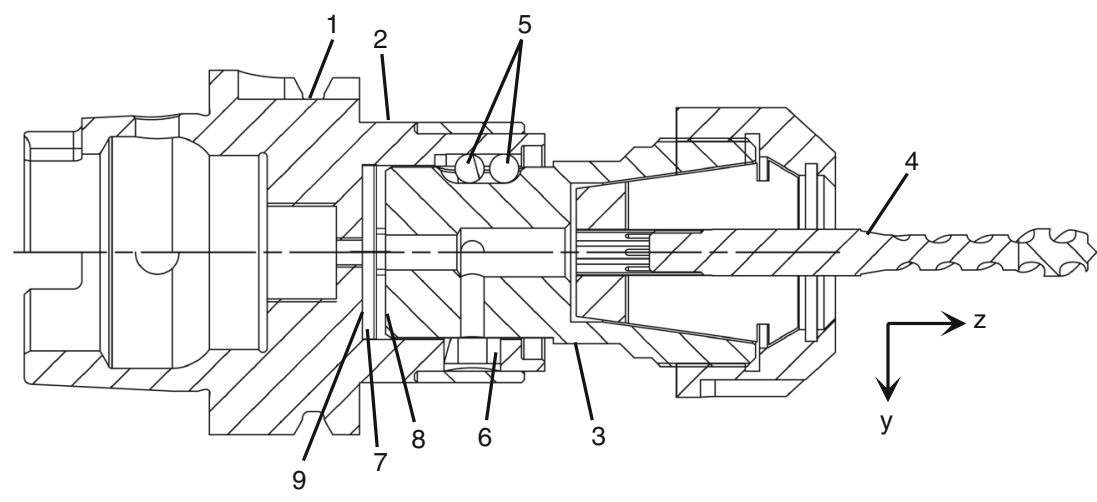


Fig. 3 Close-to-tool vibration sensor (a) with the measuring directions $a_{x}$ and $a_{y}(\mathrm{~b})$

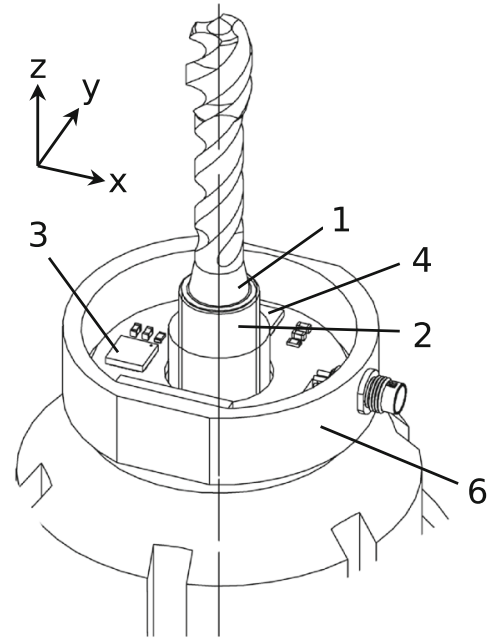

(a)

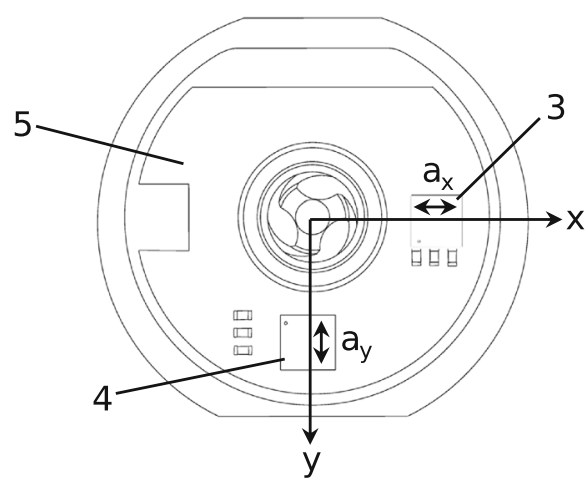

(b)
By inserting the Hooke's law $\sigma=E \epsilon$ into Eq. 2, which describes the beam stress $\sigma$ due to torque load $M_{\mathrm{L}}$,

$\sigma(y, z)=\frac{M_{\mathrm{L}}(y)}{I_{\mathrm{x}}(y)} z=\left.\frac{M_{\mathrm{L}}(y)}{I_{\mathrm{x}}(y)} \frac{t}{2}\right|_{z=\frac{t}{2}}$

yields to Eq. 3, which describes the relation of strain $\epsilon$ and torque load $M_{\mathrm{L}}$.

$\epsilon E=\frac{M_{\mathrm{L}}(y)}{I_{\mathrm{x}}(y)} \frac{t}{2}$

Furthermore, the torque load $M_{\mathrm{L}}(y)$ can be substituted by $y_{1} F_{\text {Piston }}$, where $y_{1}$ is the distance between strain gauges and the point of force application of the piston $F_{\text {Piston }}$ along the $y$-axis as shown in Fig. 6 .

Since the two springs $c_{\mathrm{p}}$ and $c_{\mathrm{s}}$, shown in Fig. 5, are connected in parallel, the piston force can be expressed by Eq. 4 ,

$F_{\text {Piston }}=\left[2 c_{\mathrm{p}}+c_{\mathrm{s}}\right] w$

where $w$ is the deflection in $z$-axis direction, thus the length compensation. By considering Eqs. 4 in Eq. 3, the equation for converting measured strain $\epsilon$ into length compensation $w$ expressed by Eq. 5 can be gathered.

$w(\epsilon)=\frac{2 E I_{\mathrm{x}}\left(y_{1}\right)}{\left[2 c_{\mathrm{p}}+c_{\mathrm{s}}\right] y_{\mathrm{l}} t} \epsilon$

with $E$ as Young's modulus, $I_{\mathrm{x}}$ as second area moment of inertia around the $x$-axis and $t$ as constant thickness of the sensor webs in $z$-axis direction.

Since the stiffness of the polymer ring elements $c_{\mathrm{p}}$ has not yet been determined, the amplified bridge voltage of the full-bridge strain gauge was used for signal analysis of the evaluation experiments in Section 6.

\subsection{Rotating telemetry unit}

A telemetry unit which is rotating with the tool holder was developed for signal processing, data acquisition and data transmission or data storage. As shown in Fig. 7 the telemetry unit is divided into two sections, where the first section is the signal preprocessing stage for the strain gauges (length compensation sensor) and the MEMS accelerometers (tool vibration sensor).
Fig. 4 Axial length compensation sensor (a) and integration into the floating tap holder Softsynchro 3 (b)

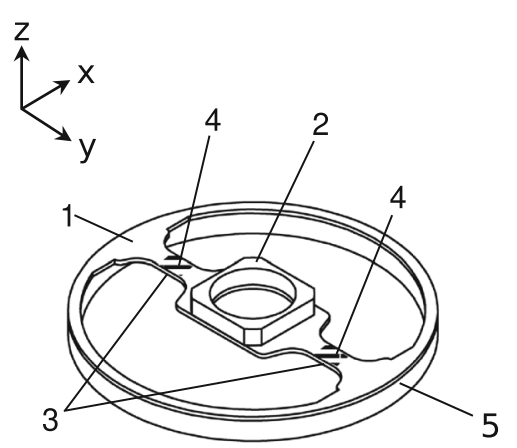

(a)

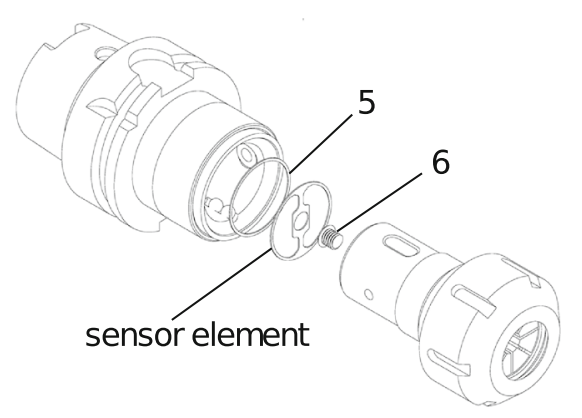

(b) 
Fig. 5 Modelling of polymer ring elements and sensor stiffness

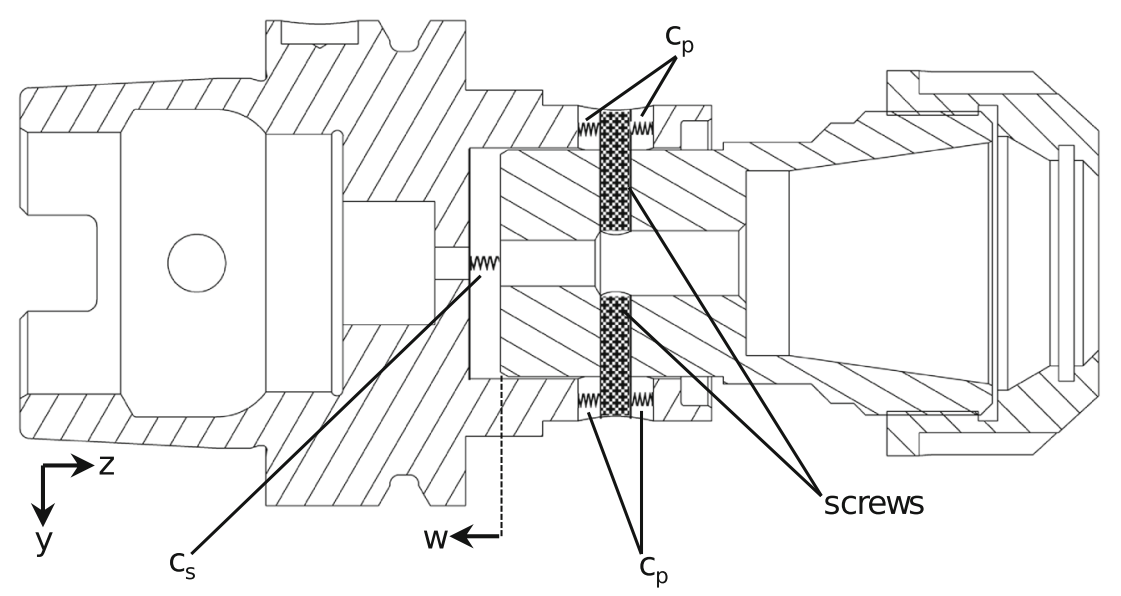

The second section includes a data acquisition stage using a 24 bit high speed four channel simultaneous analogto-digital converter (ADC) as well as a dual core embedded system running a real time operating system. The embedded system receives the bit stream of the samples from the ADC, whereby the sampling rate can be configured up to $f=$ $52.7 \mathrm{kHz}$. Depending on desired sample rate $f_{S}$, digitized samples will be transferred directly via WiFi or stored on a microSD card by the embedded system.

For attachment of the telemetry unit on the sensor-integrated floating tap holder as well as for battery integration a plastic carrier (1) shown in Fig. 8 was 3D printed.

\section{Experimental setup}

To evaluate the sensor-integrated floating tap holder, thread cutting tests of size M8 with pitch $1.25 \mathrm{~mm}$ were performed using a DMG MORI three axes vertical machining center of type DMC $850 \mathrm{~V}$.

For validation purpose, only three types of uncertainty were deliberately and randomly induced. Those were: (a) synchronization errors caused by using a pitch of $1.30 \mathrm{~mm}$ instead of $1.25 \mathrm{~mm}$ for the tapping cycle within the machine program, (b) faulty core hole diameter by using $6.6 \mathrm{~mm}$

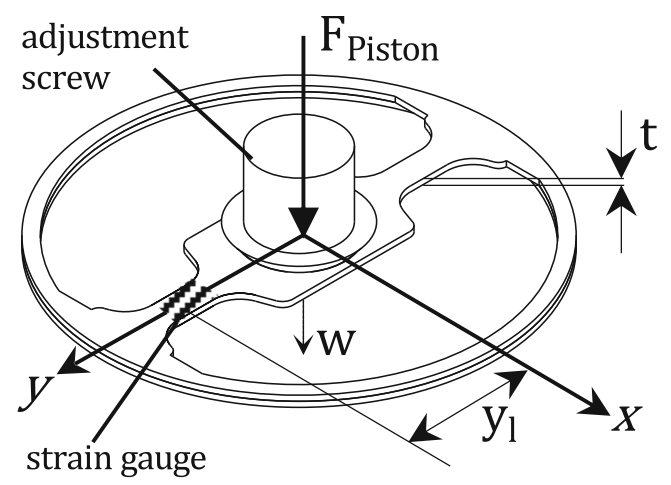

Fig. 6 Force application of the length compensation sensor and $7.0 \mathrm{~mm}$ instead of $6.8 \mathrm{~mm}$ and (c) axis offset in single axis direction of $0.3 \mathrm{~mm}$. As mentioned in Section 3 the drilling process in the first stage for manufacturing the core holes may also be afflicted with uncertainty which may affect the tapping process. To minimize uncertainty in core hole geometry during drilling, unworn solid carbide drills with internal cooling supply were used within the experiments. After the drilling process, the core holes were first flushed out with cooling lubricant to minimize the amount of the metal chips in the core holes. Then the core holes were filled with cooling lubricant fluid to provide cooling while tapping, since internal and external cooling lubricant supply could not be used, because of missing sealing of the telemetry unit. The core holes were also not chamfered after the drilling process. The workpiece material used was of type $42 \mathrm{CrMo} 4$. Furthermore, the motor spindle

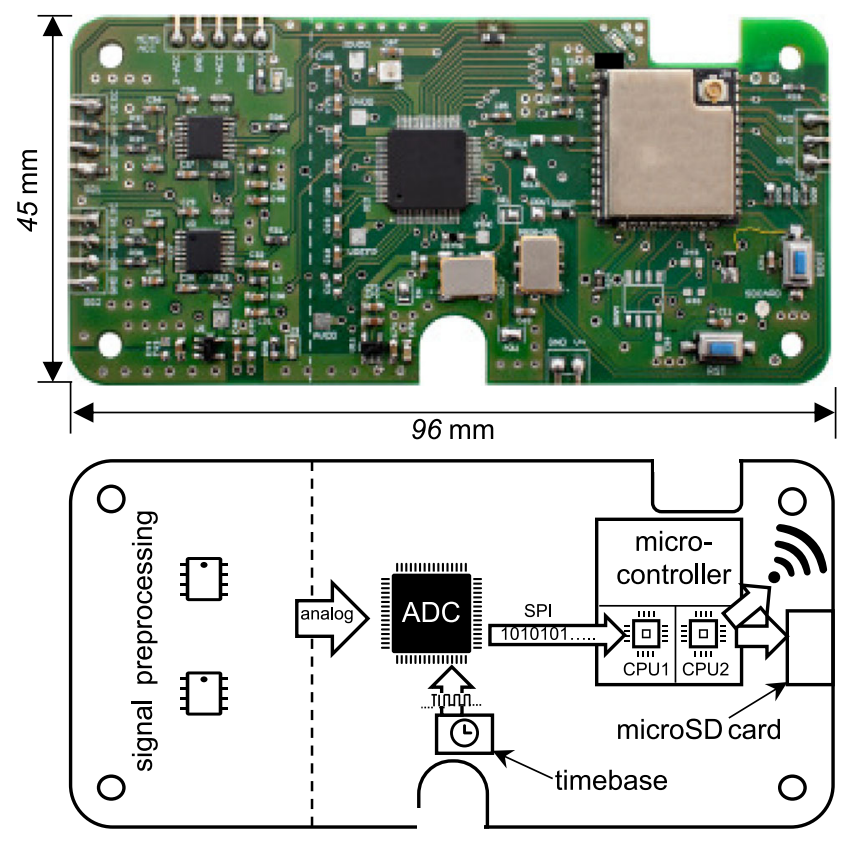

Fig. 7 Telemetry unit with main components 


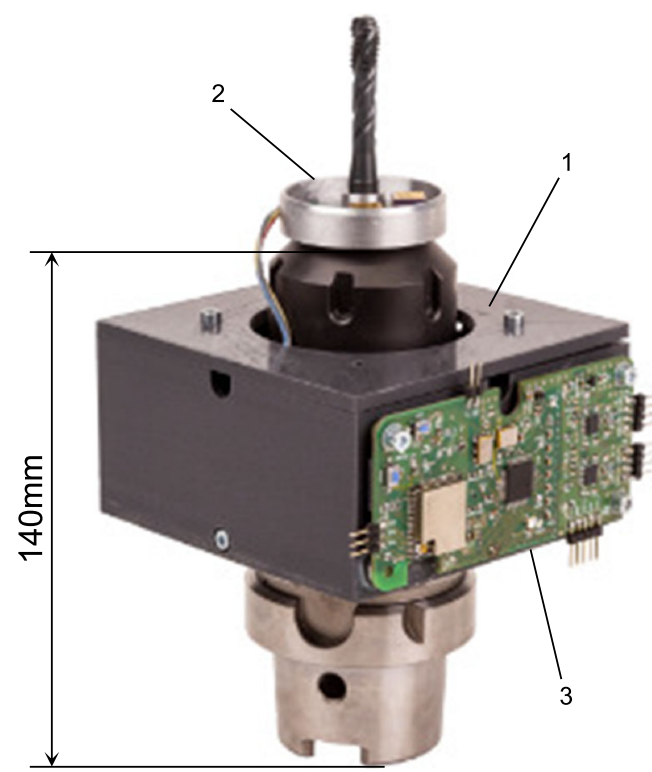

Fig. 8 Sensor-integrated floating tap holder with carrier (1), tool vibration sensor (2) and telemetry unit (3)

speed was set to $597 \mathrm{rpm}$ yielding a cutting speed of aprox. $15 \frac{\mathrm{m}}{\min }$. The tool used within the experiments was a DIN 371 M8 machine tap with cutting material of type HSS-E (HighSpeed Steel with higher cobalt content) and vaporized coating. The analog signals of the tool vibration as well as the length compensation sensor were digitized at a sample rate of $10.5 \mathrm{kHz}$, whereby the samples were transmitted via WiFi to a server application written in MATLAB for live data visualization, data storage and post analysis.

\section{Signal processing}

The integrated sensor system records the measured signals permanently resulting in a huge amount of stored sensor data. Thus, a novel approach for data preparation for smart sensor components by means of a time series signal data slicing method has been developed. The method consists of two steps:

1. Process detection: As the data acquisition system is not triggered for each manufactured thread but recorded continuously, a process detection method is necessary to separate the processes from each other. For detecting the start and the end of each tapping cycle the vibration signal measured by the tool vibration sensor is first downsampled by using a moving root mean square (rms) function with a slicing window size of 500 data points and no window overlap. The signal is then trimmed with a threshold value.

2. The cycles are not perfectly synchronized to each other, because of process variations due to varying process parameters. Therefore, a subsequent adjustment is established by applying the cross-correlation function. The first tapping cycle is considered as the reference signal while the following cycles are shifted iteratively in the time axis according to the location of the maximum correlation value.

Figure 9 shows the measured raw signals from the two MEMS accelerometers of the tool vibration sensor and the length compensation sensor for a single tapping cycle. A single tapping cylce is divided into three stages: (1) the cutting stage, (2) reversing stage with the reversal point and (3) turning out of the thread cutting tool. The segmentwise analysis revealed that the first stage contains the most information for uncertainty detection. Hence, the signals are reduced to this segment for further processing.

To generate more information content, signal transformation into different domains is applied. The time series data is transformed with the power spectral density estimation (PSD) into the frequency domain and with the short-time Fourier transformation as well as with the wavelet packet transformation (WPT) into the time-frequency domain. A more comprehensive description as well as a comparison of these two transformation methods can be found in [18]. The WPT has been applied by using the Daubechie wavelet with 4th order and decomposed into 4 levels leading to 16 nodes. Although signal transformation generates more information content, the density of information is low. Feature engineering is applied by mapping each signal to a scalar value to establish a higher information density. Different features are extracted in each domain, such as the central moments from statistics with the mean, standard deviation, skewness, and kurtosis, and further typically used features, such as rms, peak2peak, and entropy [8, 22]. Afterwards, the feature vectors are normalized by the $z$-score for better comparability. The scalar feature values can then be compared for each process to investigate if they correctly classify the different types of uncertainty considered in this work.

\section{Results}

In this section the capability of detecting different types of uncertainty during tapping with the developed sensorintegrated floating tap holder is shown.

\subsection{Axis offset and faulty core hole diameter}

The axis offset proves to be the most challenging for uncertainty detection during tapping. Only a few number of features show the potential to classify the occurrence of an axis offset. Two WPT features related to axis offset are shown in Fig. 10. 
Fig. 9 Raw signals of tool vibration ( $x$ - and $y$-axis direction) and length compensation sensor for a single tapping cycle

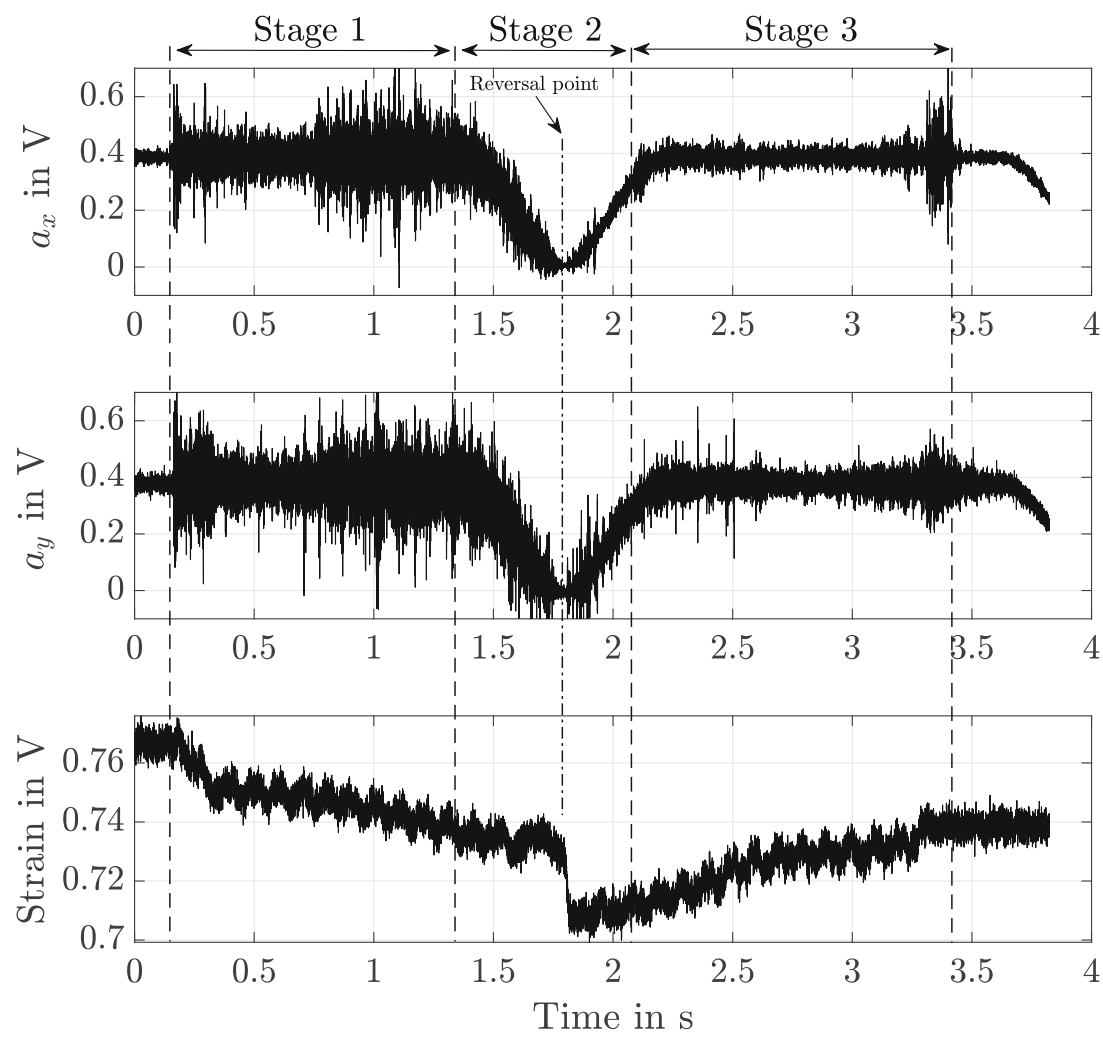

that a synchronization error caused by a pitch deviation of $+0.05 \mathrm{~mm}$ is detected by the length compensation sensor.

\subsection{Tool breakage}

Within the experiments a tapping tool breakage could be captured by the sensor-integrated floating tap holder. The tapping cycle related to the tool breakage was afflicted with all three types of uncertainty, thus axis offset of $0.3 \mathrm{~mm}$, small core hole diameter of $6.6 \mathrm{~mm}$ and synchronization error caused by pitch deviation of $+0.05 \mathrm{~mm}$. Figure 12 shows the tool vibration voltage signal in $y$-axis direction

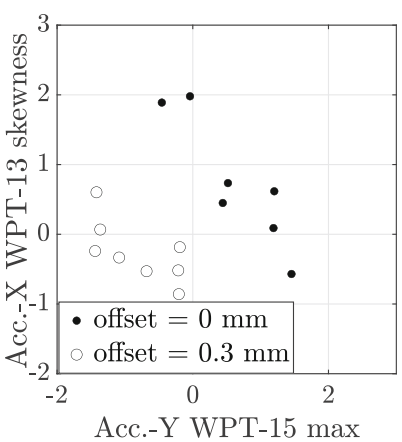

(a)

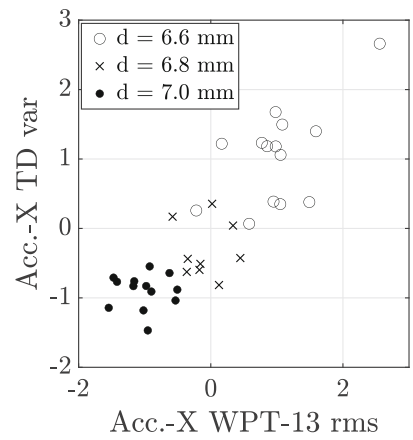

(b)
Fig. 10 Detection of uncertainty axis offset (a) and faulty core hole diameter (b) using the MEMS based tool vibration sensor 


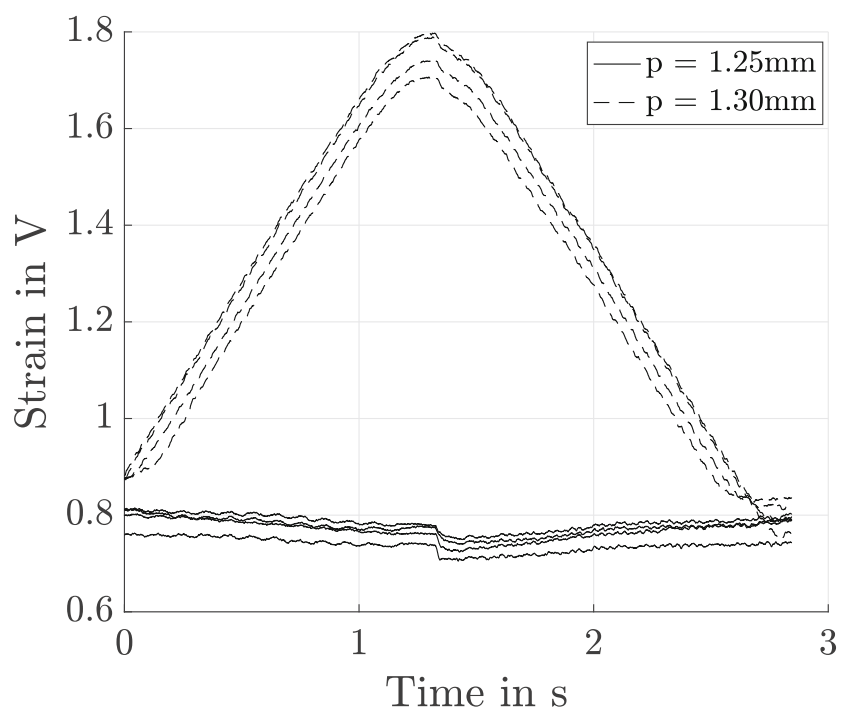

Fig. 11 Axial compensation sensor voltage signals for detecting synchronization errors. The graph shows four tapping cycles with a nominal pitch of $1.25 \mathrm{~mm}$ and for four tapping cycles with an erroneous pitch of $1.30 \mathrm{~mm}$

for multiple tapping cycles during the experiments. The vibration signal of the tapping cycles before the tool breakage occurs, clearly distinguishes from the tapping cycles after the breakage, whereby the cycle related to the tap tool breakage can be clearly seen by the high peak of approx. $\pm 1.25 \mathrm{~V}$ at the turning out stage (stage 3 according to Fig. 9).

Table 1 shows an overview of identified features which reveal to be of high importance. The detection of axis offset and faulty core hole diameter works well with tool vibration signal features derived after WPT. The synchronization error can be comprehended from the raw length compensation sensor signal so that no features must be extracted.

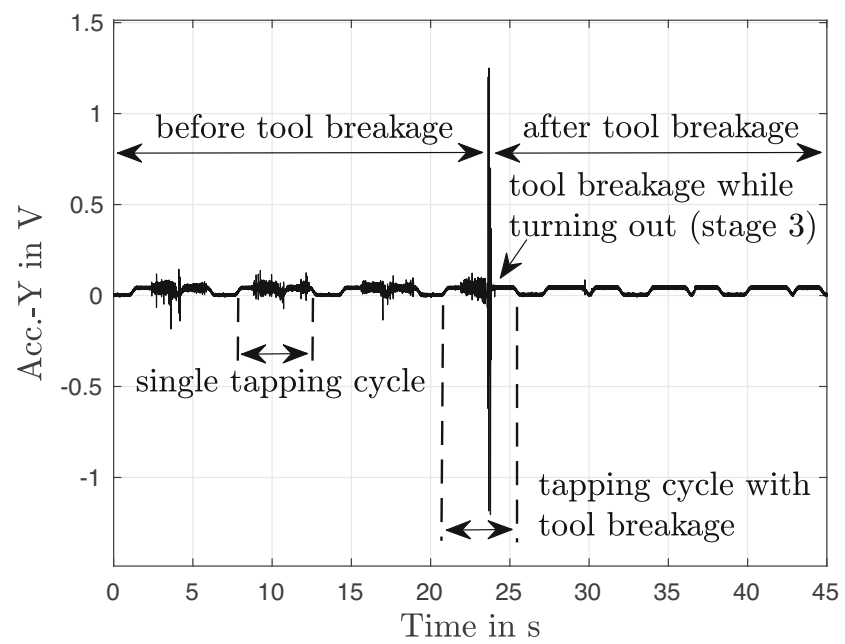

Fig. 12 Tool vibration voltage signal in $y$-axis direction for several tapping cycles with occurrence of tapping tool breakage
Table 1 Relevant features for uncertainty detection during tapping using signals of tool vibration and length compensation sensor

\begin{tabular}{lll}
\hline & Tool vibration & Length comp. \\
\hline (a) Axis offset & WPT-15 max & - \\
& WPT-13 max & \\
(b) Sloped pre-drills & - & - \\
(c) Faulty core & WPT-13 rms & - \\
hole diameter & & Time domain raw \\
$\begin{array}{l}\text { (d) Synchronization } \\
\text { error }\end{array}$ & - & - \\
Tool breakage & Time domain rms & - \\
\hline
\end{tabular}

The same also applies to tool breakage detection, whereby a sliding rms function of the tool vibration signal significantly amplifies the breakage signal.

\section{Conclusion and outlook}

This paper presents an experimental sensor-integrated floating tap holder for uncertainty detection during tapping based on tool vibration and axial length compensation measurements. Several features are extracted using feature engineering methods in the time and spectral domain and evaluated afterwards. Relevant features can be identified and mapped into a one or two dimensional representation where the tapping processes are classified into the different uncertainty groups. Two features can be extracted from the tool vibration signals to distinguish uncertainty by faulty core hole diameters with a deviation of $\pm 0.2 \mathrm{~mm}$ from the nominal core hole size of $6.8 \mathrm{~mm}$ for M8 threads. Furthermore, the axial length compensation sensor can detect and quantify synchronization errors for a M8 machine tap caused by an erroneous pitch of $1.3 \mathrm{~mm}$ for the tapping cycle within the numeric control program of the machine tool. Besides this, the tool vibration sensor is capable of detecting tool breakage within the tapping cycle. However, the example of uncertainty detection of faulty core hole diameters shows that considering only two features might be insufficient to clearly distinguish the classes from each other. Machine learning methods can be considered in further works to build enhanced approaches such as multivariate classification models that can significantly increase the classification accuracy.

For an application of the sensor-integrated floating tap holder in a real manufacturing environment, the presented system must be validated in an industrial field.

Funding Open Access funding enabled and organized by Projekt DEAL. This document contains results of the Collaborative Research 
Centre 805 (Project number 57157498) funded by the German Research Foundation (Deutsche Forschungsgemeinschaft, DFG).

Open Access This article is licensed under a Creative Commons Attribution 4.0 International License, which permits use, sharing, adaptation, distribution and reproduction in any medium or format, as long as you give appropriate credit to the original author(s) and the source, provide a link to the Creative Commons licence, and indicate if changes were made. The images or other third party material in this article are included in the article's Creative Commons licence, unless indicated otherwise in a credit line to the material. If material is not included in the article's Creative Commons licence and your intended use is not permitted by statutory regulation or exceeds the permitted use, you will need to obtain permission directly from the copyright holder. To view a copy of this licence, visit http://creativecommons. org/licenses/by/4.0/.

\section{References}

1. Ahn J, Lee D, Kim S, Kim H, Cho K (2003) Effects of synchronizing errors on cutting performance in the ultra-high-speed tapping. CIRP Ann 52(1):53-56. https://doi.org/10.1016/S00078506(07)60529-0

2. BILZ WERKZEUGFABRIK GmbH \& Co. KG (2021) HFPSystem. https://www.bilz.de/produkte/messtechnik-smarttools/ prozessueberwachung-fuergewindebearbeitung/

3. Chen YB, Sha JL, Wu SM (1990) Diagnosis of the tapping process by information measure and probability voting approach. J Eng Ind 112(4):319-325. https://doi.org/10.1115/1.2899594

4. EMUGE-Werk Richard Glimpel GmbH \& Co. KG (2021) Softsynchro3. https://www.emuge.com/sites/default/files/Emuge $\% 20$ Softsynchro\%20Brochure.pdf

5. Gil Del Val A, Fernández J, del Castillo E, Arizmendi M, Veiga F (2013) Monitoring of thread quality when tapping nodular cast iron with TiN-coated HSS cutting taps. Int J Adv Manuf Technol 69(5-8):1273-1282. https://doi.org/10.1007/s00170-013-5078-7

6. Gil Del Val A, Veiga F, Suárez A, Arizmendi M (2020) Thread quality control in high-speed tapping cycles. J Manuf Mater Process 4(1):9. https://doi.org/10.3390/jmmp4010009

7. pro-micron $\mathrm{GmbH}$ (2021) spike. https://www.pro-micron.de/wpcontent/uploads/2017/06/SPIKE-1-2-Produktbrosch\%c3\%bcre.pdf

8. Guo W, Li B, Shen S, Zhou Q (2019) An intelligent grinding burn detection system based on two-stage feature selection and stacked sparse autoencoder. Int J Adv Manuf Technol vol 103. https://doi.org/10.1007/s00170-019-03748-5

9. Lee DJ, Kim SH, Ahn JH (2004) Breakage detection of smalldiameter tap using vision system in high-speed tapping machine with open architecture controller. KSME Int J 18(7):1055-1061. https://doi.org/10.1007/BF02983280

10. Li W, Li D, Ni J (2003) Diagnosis of tapping process using spindle motor current. Int $\mathrm{J}$ Mach Tools Manuf 43:73-79. https://doi.org/10.1016/S0890-6955(02)00142-6

11. Liu T, Ko E, Sha S (1991) Diagnosis of tapping operations using an AI approach. In: Proceedings 1991 IEEE international conference on robotics and automation. IEEE Comput. Soc. Press, Sacramento, CA, USA, pp 1556-1561. https://doi.org/10.1109/ROBOT. 1991.131838

12. Liu TI, Lee J, Liu G, Wu Z (2013) Monitoring and diagnosis of the tapping process for product quality and automated manufacturing. Int J Adv Manuf Technol 64(5-8):1169-1175. https://doi.org/10. 1007/s00170-012-4058-7

13. Ma YC, Wan M, Yang Y, Zhang WH (2019) Dynamics of tapping process. Int J Mach Tools Manuf 140:34-47. https://doi.org/10. 1016/j.ijmachtools.2019.02.002

14. ARPOSS Monitoring Solutions GmbH (2021) DDU SENSOR SYSTEM. https://www.marposs.com/media/5090/d-1/t-file/ ARTIS_DB_DDU_SENSOR_SYSTEM_EN.pdf

15. Moehring HC, Werkle K, Maier W (2020) Process monitoring with a cyber-physical cutting tool. Procedia CIRP 93:1466-1471. https://doi.org/10.1016/j.procir.2020.03.034

16. Monka P, Monkova K, Uban M, Hruzik L, Vasina M (2018) Vibrodiagnostics as the tool of a tap wear monitoring. Procedia Struct Integr 13:959-964. https://doi.org/10.1016/j.prostr.2018.12.179

17. Polvorosa R, de Lacalle LL, Egea AJS, Fernandez A, Esparta M, Zamakona I (2020) Cutting edge control by monitoring the tapping torque of new and resharpened tapping tools in Inconel 718. Int J Adv Manuf Technol 106(9-10):3799-3808. https://doi. org/10.1007/s00170-019-04914-5

18. Sauter E, Sarikaya E, Winter M, Wegener K (2021) Inprocess detection of grinding burn using machine learning. Int J Adv Manuf Technol pp 1-17. https://doi.org/10.1007/s00170021-06896-9

19. Gil del Val A, Fernández J, Diéguez PM, Arizmendi M, Veiga F (2014) Real time diagnosis charts of thread quality in tapping operations. Mater Sci Forum 797:71-77. https://doi.org/10.4028/ www.scientific.net/MSF.797.71

20. VDI (2015) VDI 3334 Blatt 1 (2008-11): Machining of internal threads. Beuth Verlag, Berlin

21. Wang C, Cheng K, Chen X, Minton T, Rakowski R (2014) Design of an instrumented smart cutting tool and its implementation and application perspectives. Smart Mater Struct vol 23. https://doi.org/10.1088/0964-1726/23/3/035019

22. Yuqing Z, Xue W (2018) A multisensor fusion method for tool condition monitoring in milling. Sensors 18:3866. https://doi.org/ $10.3390 / \mathrm{s} 18113866$

Publisher's note Springer Nature remains neutral with regard to jurisdictional claims in published maps and institutional affiliations. 